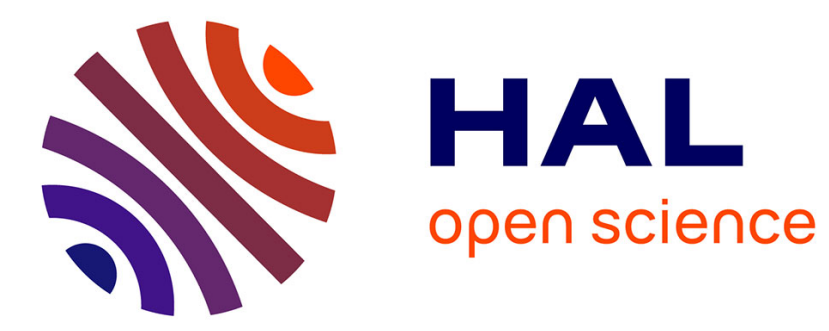

\title{
Marcel Duchamp : trois méthodes pour mettre le hasard en conserve
}

\author{
Sarah Troche
}

\section{To cite this version:}

Sarah Troche. Marcel Duchamp : trois méthodes pour mettre le hasard en conserve. Cahiers philosophiques, 2012, $\mathrm{n}^{\circ} 131$ (4), pp.18. 10.3917/caph.131.0018 . hal-03145967

\section{HAL Id: hal-03145967 \\ https://hal.science/hal-03145967}

Submitted on 18 Feb 2021

HAL is a multi-disciplinary open access archive for the deposit and dissemination of scientific research documents, whether they are published or not. The documents may come from teaching and research institutions in France or abroad, or from public or private research centers.
L'archive ouverte pluridisciplinaire HAL, est destinée au dépôt et à la diffusion de documents scientifiques de niveau recherche, publiés ou non, émanant des établissements d'enseignement et de recherche français ou étrangers, des laboratoires publics ou privés. 


\title{
MARCEL DUCHAMP : TROIS MÉTHODES POUR METTRE LE HASARD EN CONSERVE
}

\author{
Sarah Troche
}

Réseau Canopé | «Cahiers philosophiques »

$2012 / 4 \mathrm{n}^{\circ} 131$ | pages 18 à 36

ISSN 0241-2799

Article disponible en ligne à l'adresse :

https://www.cairn.info/revue-cahiers-philosophiques1-2012-4-page-18.htm

Distribution électronique Cairn.info pour Réseau Canopé.

(C) Réseau Canopé. Tous droits réservés pour tous pays.

La reproduction ou représentation de cet article, notamment par photocopie, n'est autorisée que dans les limites des conditions générales d'utilisation du site ou, le cas échéant, des conditions générales de la licence souscrite par votre établissement. Toute autre reproduction ou représentation, en tout ou partie, sous quelque forme et de quelque manière que ce soit, est interdite sauf accord préalable et écrit de l'éditeur, en dehors des cas prévus par la législation en vigueur en France. Il est précisé que son stockage dans une base de données est également interdit. 


\section{Marcel Duchamp}

\section{MARCEL DUCHAMP : \\ TROIS MÉTHOdES POUR METTRE LE HASARD EN CONSERVE}

Sarah Troche

Si le hasard est, dès 1913, un acteur à part entière de la production de Duchamp, il semble difficile d'en parler de manière générale, indépendamment des mécaniques ingénieuses inventées par l'auteur pour le " mettre en conserve ». Car le hasard, loin d'être un symbole de la chance ou de l'irrationnel, est avant tout une opération de pensée, qui se réinvente dans chaque œuvre à travers des méthodes différentes. Ainsi l'Erratum musical permet-il de questionner la notion d'empreinte mémorielle, les formes aléatoires des 3 stoppages étalon érodent la norme du mètre par excès de précision, et l'Obligation pour la roulette de Monte-Carlo transforme la martingale en jeu de patience. En portant une attention précise aux opérations de hasard spécifiques à chaque œuvre ainsi qu'aux mouvements de pensée qui les accompagnent, nous verrons comment ces objets se font écho en élaborant, chacun à leur manière, une esthétique suspensive.

armi les nombreux portraits dont Marcel Duchamp a fait l'objet, celui que dresse André Breton dans Les Pas perdus illustre à merveille ce « dédain de la thèse ${ }^{1} »$ qui permet à l'œuvre de Duchamp d'échapper à toute affiliation directe comme aux explications systématiques. Ce portrait, tout en creux et en contournements, est d'abord celui d'un « état d'esprit », que Breton saisit à travers un geste aléatoire qu'il qualifie d'« extraordinaire » : 
J'ai vu faire à Duchamp une chose extraordinaire, jeter en l'air une pièce en disant : «Pile je pars ce soir en Amérique, face je reste à Paris. » À cela nulle indifférence, il préférait sans doute infiniment partir, ou rester. Mais la personnalité du choix, dont Duchamp est un des premiers à avoir proclamé l'indépendance en signant, par exemple, un objet manufacturé, n'est-elle pas la plus tyrannique de toutes et ne convient-il pas de la mettre à cette épreuve, pourvu que ce ne soit pas pour lui substituer un mysticisme du hasard ${ }^{2}$ ?

À travers cette description, Breton définit précisément le point d'articulation entre l'intervention du hasard et l'indifférence légendaire de Duchamp : il ne dit pas que Duchamp recourt au hasard parce qu'il n'a pas de préférence et que peu lui importe d'être ici ou ailleurs ; si tel était le cas, le hasard serait le prolongement de la "liberté d'indifférence » que Descartes qualifie, dans les Méditations métaphysiques, de "plus bas de degré de la liberté ${ }^{3}$ », celle qui s'exerce en l'absence de raisons et de connaissances. Au contraire, nous dit Breton, Duchamp «préférait sans doute infiniment partir ou rester $»$ : recourir au hasard est une façon, non de traduire une indifférence préalable, mais de suspendre activement le jeu des préférences, de mettre à l'épreuve "la personnalité du choix », qui est "la plus tyrannique de toutes ». En soumettant la décision au hasard, il

Le recours au hasard ne s'avère efficace que lorsqu'il est dépouillé de toute projection subjective s'agit donc de déplacer le principe du choix et de substituer, aux hiérarchies préférentielles et aux inclinations du goût, l'équiprobabilité d'un tirage au sort. Mais cette logique suspensive ne peut avoir d'efficace, précise Breton, qu'à condition de ne pas teinter le jeu de pile ou face d'un «mysticisme du hasard»: s'en remettre au hasard pourrait être une façon superstitieuse de rejoindre la croyance au destin, qui n'efface pas la « personnalité du choix », mais l'englobe et la justifie au sein d'un ordre supérieur, inaccessible à la seule raison. Le recours au hasard ne s'avère efficace que lorsqu'il est dépouillé de toute connotation superstitieuse, et donc de toute projection subjective. C'est aussi ce qui le rend «extraordinaire » aux yeux de Breton : à l'encontre du hasard surréaliste, qui noue étroitement le désir subjectif au monde extérieur dans les rencontres poétiques et les "pétrifiantes coïncidences ${ }^{4} »$, le hasard de Duchamp permet de s'affranchir de la subjectivité en suspendant méthodiquement la norme du goût.

Cette citation de Breton pose les enjeux de l'étude du hasard chez Duchamp : contre toute lecture systématique, qui verrait dans le hasard le prolongement de l'indifférence de Duchamp ou le symbole d'un art éminemment paradoxal, il faut penser son caractère opératoire. Le hasard est un moyen, et non une fin en soi qui serait sacralisée comme figure de

2. Ibid., p. 119-120. Les italiques sont de l'auteur.

3. René Descartes, Méditations métaphysiques, Méditation IV, Paris, PUF, 1996, p. 88.

4. André Breton, Nadja, Paris, Gallimard, 1964, p. 20. 
l'irrationnel ou de l'absence de choix. Il ne peut être compris qu'au sein des mécanismes et des opérations précises qui lui confèrent une efficacité suspensive. C'est pourquoi il nous paraît nécessaire d'ancrer notre étude dans l'analyse aussi détaillée que possible des œuvres intégrant le hasard, afin de saisir sa signification au regard de son fonctionnement dans chaque dispositif d'ensemble.

Nous nous concentrerons sur les trois interventions principales du hasard dans l'œuvre de Duchamp : 3 stoppages étalon (1913), Erratum musical (1913) et Obligation pour la roulette de Monte-Carlo (1924). L'attention que leur a accordée la postérité est inégale : les analyses fort nombreuses consacrées aux 3 stoppages étalon, symbole de la « chute » des normes esthétiques et de l'effacement de la subjectivité de l'auteur, sont sans commune mesure avec ces objets plus discrets que sont Obligation pour la roulette de Monte-Carlo et les partitions musicales. Dans ces trois objets, le hasard est indissociable d'une mécanique précise : il n'a pas de signification intrinsèque, il ne fait sens qu'articulé au titre de l'œuvre, au texte éventuel et aux opérations de pensée qui sous-tendent le geste aléatoire. Tout en maintenant l'exigence d'une attention spécifique à l'usage $\mathrm{du}$ hasard dans chaque contexte, nous verrons comment ces objets se font écho et élaborent, chacun à leur manière, une esthétique suspensive : par le recours méthodique au hasard, la répétition ne produit nulle « empreinte », la succession des tirages ne marque aucune progression, la précision des formes singulières et des combinaisons aléatoires met en échec toute approche normative.

\section{3 stoppages étalon : un protocole expérimental de "l'inframince "}

Les 3 stoppages étalon ont été réalisés en plusieurs étapes : en $1913-$ 1914, Duchamp écrit une note détaillant le protocole (« L’idée de la fabrication »), puis applique le procédé (lâcher trois fils d'un mètre de long, à un mètre de hauteur, sur une toile peinte en bleu) ; en 1918, il découpe et met sous verre les bandes de toile sur lesquelles les trois fils sont collés, et réalise trois règles en bois épousant les linéaments des fils de manière à pouvoir les reproduire, ultérieurement, sur d'autres supports ; en 1953, il ajoute deux autres règles droites, d'un mètre de long, pour « clarifier le protocole expérimental ». "L'idée de la fabrication »sera partiellement reproduite dans La Boîte verte de 1934, qui regroupe les croquis, notes et études relatives au processus de fabrication du Grand Verre; cette note apparaît sous la rubrique «Hasard », précédée de la mention suivante : « 3 stoppages étalon, Du hasard en conserve. $1914^{5} »$. Les trois fils, tels qu'ils sont exposés au regard du spectateur, sont indissociables de l'opération présidant aux résultats : présentés au sol («Ce n’est pas un tableau $\left.{ }^{6} »\right)$, ils se découvrent au regard «stoppés » dans leur chute. Le résultat aurait été tout autre s'ils avaient été exposés au mur, comme une toile : on aurait alors contemplé, non le produit d'une opération, mais l'écriture particu-

5. Marcel Duchamp, "La Boîte verte », in Duchamp du signe, Paris, Flammarion, 1994, p. 50.

6. "À propos de moi-même ", in Duchamp du signe, op. cit., p. 224. 
Marcel Duchamp, 3 stoppages étalon, 1913, $28 \times 129 \times 23 \mathrm{~cm}$, Paris, musée national d'Art moderne - Centre Pompidou.

lière des trois formes sinueuses. Les trois bandes bleues recevant les trois fils sont de tailles et de couleurs identiques, mettant ainsi en relief deux dimensions contraires : l'interchangeabilité des mètres et la singularité de leurs mouvements ondulatoires. Cette tension se retrouve dans les règles en bois, qui ont l'aspect paradoxal d'outils de mesure non normatifs. S'il y a un sens à décrypter, celui-ci n'a pas l'opacité du mystère, mais invite le spectateur à articuler ensemble les pièces de cette mécanique pour faire surgir les significations en « associant » une idée à une opération, un geste à une loi, une mesure à sa déformation. 
Si Duchamp choisit un élément aussi souple et fin que le fil, de préférence à des éléments beaucoup plus maniables (des baguettes, des jetons, des boules), c'est bien, comme le montre Maurice Fréchuret, pour les circonvolutions exactes que cette souplesse permet, les effets de « déformation » dans la chute, le louvoiement de la forme serpentine ${ }^{7}$. Mais la description de ces formes dessinées par le hasard doit cependant tenir compte d'une caractéristique manifeste : les fils sont, à l'observation, étrangement peu déformés et peu expressifs. Ce constat a motivé une investigation menée par Rhonda R. Shearer et Stephen J. Gould, qui ont méthodiquement réitéré le processus mis en place par Duchamp ${ }^{8}$. Or l'expérience montre qu'un fil d'un mètre de long lâché au-dessus d'un support horizontal ne se rapproche jamais des formes des 3 stoppages étalon, qui présentent une régularité et une absence de sinuosité trop importantes pour ne pas avoir fait l'objet d'une intention. Les deux auteurs ont donc soutenu la thèse suivante : Duchamp aurait, contrairement à ce qu'il a toujours affirmé, corrigé les résultats du hasard en manipulant les trois fils. En examinant les 3 stoppages étalon, il apparaît également que les fils traversent la toile à chaque extrémité, qu'ils mesurent plus d'un mètre et n'ont pas été collés, mais cousus, en conformité avec le terme de «stoppage », qui désigne un raccommodage permettant de «stopper » une déchirure. Cette thèse a été contestée et le débat ne sera sans doute jamais tranché 9 . Quand bien même le résultat serait légèrement corrigé (comme certains ready-made sont «assistés » ou « rectifiés»), on ne saurait voir dans 3 stoppages étalon une expérience falsifiée, qui chercherait à duper le spectateur. Il faut donc, quelle que soit la nature exacte du processus, s'en tenir à la réalité de ce qui nous est présenté : les lignes sont étrangement peu expressives et pourtant différentes les unes des autres ; la neutralité de l'ensemble est donc aussi importante que l'absolue singularité de chaque ligne isolée.

Que peut signifier cette neutralité, arrangée ou non, tout du moins manifeste ? Ces trois lignes, paradoxalement, ne nous évoquent rien, alors qu'il y aurait lieu de leur associer mille et une références et images. Ainsi Didier Semin retrace, d'Albrecht Dürer à Sigmar Polke, la « polysémie fabuleuse » de la ligne serpentine, «forme ouverte » par excellence, pour souligner, par la négative, l'incongruité de l'absence d'expressivité des trois lignes de Duchamp ${ }^{10}$. Si l'hypothèse d'un « accommodement duchampien avec le hasard » ne peut être plus qu'une hypothèse, elle n'est pas sans cohérence au regard de la démarche de Duchamp ; car il est probable,

7. Maurice Fréchuret, "Trois stoppages étalon », Le Mou et ses formes : essai sur quelques catégories de la sculpture du Xxe siècle, Nîmes, Jacqueline Chambon, 2004, p. 27-44.

8. Rhonda R. Shearer et Stephen J. Gould, « Hidden in Plain Sight: Duchamp's 3 Standard Stoppages. More Truly a "Stoppage" (An Invisible Mending) Than We Ever Realized », disponible sur le site : Tout-Fait: The Marcel Duchamp Studies Online Journal, n 1, 1999 (http://www.toutfait.com).

9. Herbert Molderings considère que l'argumentation ne peut être validée sur le plan technique : " Si I'on durcit un fil retors fin en le passant au bloc de cire d'abeille - méthode employée par les relieurs et les tailleurs spécialistes du cuir - et qu'on réalise l'expérience de la chute des fils décrite par Duchamp, on obtient une incurvation peu prononcée, semblable à celle des 3 stoppages étalon " (Herbert Molderings, L'Art comme expérience : les 3 stoppages étalon de Marcel Duchamp, Paris, Maison des sciences de l'homme, 2007, p. 46).

- 10. Didier Semin, "La ligne du célibat. Le hasard, I'arabesque et la volute : pour servir à une histoire du zigloogloo », in La Piste du hérisson, Nîmes, Jacqueline Chambon, 2004, p. 213-241. 
comme le suggère Semin, que Duchamp a neutralisé les ondulations de la ligne afin de «stopper » les images qu'elles pourraient convoquer : «Un hasard trop généreux - il a bien entendu son charme, mais celuilà, Marcel Duchamp le laissera au surréalisme de Breton - aurait pu se voir prescrire, dans le monde duchampien, une opération de chirurgie anti-esthétique ${ }^{11}$. » Le passage de la conception à la réalisation élimine ce qui était mentionné à la fin des premières notes des 3 stoppages étalon, et qui ne sera ensuite plus jamais repris : "Faire un tableau de hasard heureux ou malheureux (veine ou déveine ${ }^{12}$ ). » Impossible de voir en effet, dans ces trois lignes peu serpentines, le signe d'un bonheur ou d'un malheur quelconque, d'une réussite ou d'un échec ; le caractère non expressif annule toute projection sur ces lignes qui n'écrivent rien, si ce n'est trois formes possibles. Là où le « hasard objectif » de Breton noue de manière troublante l'inconscient de l'individu à la réalité des événements, le hasard de Duchamp affirme de façon manifeste son extériorité à toute approche subjective ${ }^{13}$.

Le chiffre trois, que l'on retrouve dans Erratum musical (trois partitions pour trois voix), participe à cette neutralité de l'ensemble : «Pour moi le chiffre trois a une importance mais pas du tout du point de vue ésotérique, simplement du point de vue numération : un, c'est l'unité, deux c'est le double, la dualité, et trois c'est le reste ${ }^{14}$. » Présenter un seul fil concentrerait le regard sur «l'unité » de l'objet, sur son expressivité particulière ; deux fils introduiraient des effets de comparaison immédiats, entre symétrie et dissymétrie (la « dualité ») ; la présence de trois fils ouvre sur « le reste », le domaine du purement factuel. Les 3 stoppages étalon exposent trois formes contingentes, précises et indéterminées à la fois, ou, comme le dit Duchamp dans une note, simplement « possibles ${ }^{15}$ », et efficaces par cette simplicité même. Les trois fils viennent « figurer » l'aspect corrosif du simplement possible, en articulant la «figuration » d'une possibilité singulière à une norme représentée dans son sens le plus concret : le mètre, la règle, la mesure - et à travers elle, l'ordre et l'harmonie. On ne peut, bien évidemment, considérer ces trois fils stoppés dans leur chute indépendamment du titre et des règles en bois qui les accompagnent : le sens, récusé par l'inexpressivité des lignes, ressurgit dans la mise en rapport des éléments ; car c'est au spectateur d'activer mentalement la machine, « d'associer l'idée d'un fil droit horizontal à celle de sa propre déformation, à son gré16 », donc de faire surgir les échos entre le mètre en bois épousant la forme des fils, l'idée de chute, et les linéaments neutres et pourtant singuliers. Le possible ne s'oppose pas à l'étalon, mais « mord » sur celui-ci, en couplant l'idée de mètre à

11. Ibid., p. 234.

12. "La Boîte de 1914 ", in Duchamp du signe, op. cit., p. 36.

- 13. Il en est de même pour les ready-made, dont le choix est tout autre que le désir précipité sur la " trouvaille » surréaliste.

14. Pierre Cabanne, Entretiens avec Marcel Duchamp, Paris, Pierre Belfond, 1967, p. 81.

- 15. "Le possible est seulement / un " mordant" physique [genre vitriol] / brûlant toute esthétique ou callistique » (" Possible », in Duchamp du signe, op. cit., p. 104).

16. Pierre Cabanne, Entretiens avec Marcel Duchamp, op. cit., p. 81. Nous soulignons. 
sa réalité physique. Les 3 stoppages étalon viennent donc assouplir le vecteur de référence, biaiser les formes et « jeter un doute pataphysique sur le concept selon lequel la droite est le plus court chemin d'un point à un autre ${ }^{17}$ ». «Un doute », seulement un doute : les 3 stoppages étalon sont, non des condamnations, mais des points d'interrogation sinueux, des soupçons devant la vérité et l'immuabilité de toute norme, qu'elle soit esthétique (ce qui est art et ce qui ne l'est pas, ce qui est goût ou non), scientifique ou politique ${ }^{18}$.

Les croisements avec La Science et l'Hypothèse (1902) d'Henri Poincaré, que Duchamp avait lu, ont fait l'objet de nombreuses analyses, qui s'appuient sur des lectures minutieuses. Les méandres du mètre et le doute jeté sur l'idée que « la droite est le plus court chemin d'un point à un autre » sont généralement mis en rapport avec le développement des géométries non euclidiennes et le « conventionnalisme » de Poincaré19. Duchamp applique, en les prenant à rebours, les principes de Poincaré : alors que ce dernier met en évidence l'impossibilité de traduire expérimentalement les conventions géométriques, et conséquemment le nonrecoupement de l'espace idéal avec la réalité physique, Duchamp accouple ces deux dimensions et transforme le mètre géométrique en ce corps particulièrement souple qu'est le fil : «En la traitant [la droite idéale] comme un corps solide, un fil en l'occurrence, en la soumettant au test de la réalité des lois physiques, il pratique la géométrie comme une science expérimentale, ce que, par définition, elle n'est pas ${ }^{20}$. » Les 3 stoppages étalon proposent donc une application sinueuse de la pensée de Poincaré, qu'ils ne viennent pas directement « illustrer », mais qu'ils prennent à l'envers, épousant le mouvement retors d'un raisonnement par l'absurde en figurant les conséquences d'une impossibilité. Le couplage discordant entre le mètre et le fil renvoie indirectement la mesure idéale et la réalité physique à leur statut de « célibataire ».

Le « test de réalité » que proposent les 3 stoppages étalon nous semble cependant fonctionner à deux niveaux : il rejoint par l'absurde le conventionnalisme de Poincaré, mais il donne aussi une forme visible aux causes infimes qui interviennent dans la chute de trois fils et qui déterminent la forme particulière du «stoppage ». Le chiffre trois annule toute dualité, tout effet de comparaison pour faire surgir l'absolue singularité (non expressive) du résultat. La répétition du même geste, effectué trois fois

17. Marcel Duchamp, "À propos de moi-même ", in Duchamp du signe, op. cit., p. 225.

- 18. Bernard Marcadé rappelle la dimension politique du mètre à l'époque de la création des 3 stoppages étalon: « En 1913, le mètre-étalon possède un statut éminemment politique puisqu'un décret de 1882 interdit d'employer en France des unités autres que les unités légales pour la mesure des grandeurs, dans les domaines de l'économie, de la santé et de la sécurité publique ainsi que dans les opérations à caractère administratif » (in Marcel Duchamp, Paris, Flammarion, p. 93).

- 19. Cf. Craig E. Adcock, "Conventionalism in Henri Poincaré and Marcel Duchamp », Art Journal, vol. XLIV, $\mathrm{n}^{\circ} 3,1984$, p. 249-258; Gerald Holton, "Henri Poincaré, Marcel Duchamp and Innovation in Science and Art ", Leonardo, vol. XXXIV, n², 2001, p. 127-134. Voir aussi le chapitre v, "Application humoristique de la géométrie non euclidienne ", in Herbert Molderings, L'Art comme expérience, op. cit., p. 109-125.

20. Ibid., p. 55. Herbert Molderings dresse également un rapprochement avec les analyses que Poincaré consacre à " l'école du fil " dans le chapitre "La mécanique classique " de La Science et l'Hypothèse : "Parmi les descriptions caractéristiques de cette "école du fil", nombre de constellations peuvent avoir inspiré Duchamp » (ibid., p. 56). 
de suite, prend ici tout son sens : le geste de lâcher le fil aura beau être parfaitement codifié et mécanisé de manière à s'effectuer de la façon la plus précise possible (sans le « hasard de la main»), le résultat sera chaque fois différent. Il n'y a là

Le chiffre trois annule toute dualité, tout effet de comparaison pour faire surgir l'absolue singularité (non expressive) du résultat aucune tension ou effet de contradiction entre le geste (le même) et les résultats (différents) : bien au contraire, la répétition exacte est la condition du sens de l'opération. C'est parce que le geste est répété à l'identique, aussi mécanique que possible, que les différences d'un fil à l'autre peuvent mettre en évidence la part non maîtrisable des causes qui interviennent à chaque lâcher : l'atmosphère du lieu, les courants d'air infimes, mais aussi les micromouvements inconscients de la main, la pression des doigts, le positionnement du corps, l'intensité de l'énergie musculaire.

C'est bien à ce niveau, nous semble-t-il, que le rapprochement avec Poincaré est le plus éclairant. Au chapitre IV de Science et Méthode (1908), intitulé « Le hasard», Poincaré expose une approche radicalement nouvelle de ce concept. Le hasard n'est pas l'autre nom de notre ignorance, toujours relative et variable en fonction des situations, mais il correspond à certains types de phénomènes que l'on peut décrire objectivement et vis-à-vis desquels la connaissance ne peut être qu'approximative et probabiliste : un phénomène peut être dit objectivement fortuit lorsqu'il est déterminé par un ensemble de causes fines et/ou complexes dont les variations les plus minimales produisent des écarts ou des différences importantes dans les résultats. En conséquence, il suffit de dévier très légèrement une des données initiales pour que le résultat soit tout autre. Au centre du phénomène fortuit se trouve donc le constat d'une disproportion évidente entre les causes et les effets, qui soustrait l'événement à toute prédiction possible : «Une cause très petite, qui nous échappe, détermine un effet considérable que nous ne pouvons pas ne pas voir ${ }^{21}$. » Poincaré distingue ainsi plusieurs types de phénomènes fortuits, selon que dominent, dans le processus, les variations infimes à l'origine du résultat (l'impulsion que je donne à la roulette d'un jeu de hasard, geste simple dont je ne peux apprécier les microvariations musculaires, décide du caractère noir ou rouge, perdant ou gagnant du résultat ${ }^{22}$ ), leurs interactions (la complexité des interactions causales à l'origine de la distribution des gouttes sur un pavé ou des molécules d'un gaz ${ }^{23}$ ) ou l'indépendance des séries d'événements (comme dans l'exemple fameux de la chute d'une tuile sur la tête d'un passant, en reprenant le modèle explicatif d'Antoine-Augustin Cournot ${ }^{24}$ ). Les 3 stoppages étalon pourraient très bien s'inscrire à la suite de cette série d'exemples donnés par Poincaré : comme les poussières

21. Henri Poincaré, Science et Méthode, Paris, Flammarion, 1947, p. 68. Nous soulignons.

22. Ibid., p. 70-71.

23. Ibid., p. 73-74.

24. Ibid., p. 77-78. 
flottant à la surface d'un vase ou les gouttes d'eau sur un pavé, les écarts d'une ligne à l'autre manifestent les variations infimes des causes ayant présidé au résultat. La «sensibilité aux conditions initiales » mise en évidence par Poincaré dans les phénomènes fortuits pourrait se dire, en termes duchampiens, sensibilité « infra mince »- l'inframince relevant de l'écart infinitésimal qui sépare deux objets, deux sensations, deux situations infiniment proches et pourtant distinctes ${ }^{25}$.

On pourrait ainsi reprendre les exemples précédents en les classant autrement, en fonction non de la complexité des causes mais du rapport de l'individu au phénomène fortuit : hasard observé dans les phénomènes naturels (imprédictibilité des variations climatiques, répartition des gouttes sur un pavé), hasard rencontré, heureux ou malheureux (la tuile), hasard artificiellement fabriqué (le jeu de hasard, comme la roulette ou le lancer de dés, permettant de reproduire à volonté la disproportion entre des causes non maîtrisables et des effets décisifs), et enfin hasard artificiellement représenté, saisi et conservé par un processus de «figuration » (3 stoppages étalon mais aussi L'Élevage de poussière [1920], la photographie prise par Man Ray faisant ressortir le relief imprédictible des poussières accumulées pendant trois mois sur la partie inférieure du Grand Verre ${ }^{26}$ ). Le hasard, tel que défini par Poincaré, est bien un acteur à part entière de l'opération des 3 stoppages étalon, mais aussi le sujet de cette œuvre : les ondulations des fils agissent comme un révélateur des microcauses intervenant dans l'événement fortuit sur le mode de "l'étant donné » (étant donné la main de Duchamp à tel instant dans telle pièce lâchant tel fil d'un mètre de long à un mètre de hauteur). Les 3 stoppages étalon peuvent être pensés comme un mécanisme de figuration de ces causes inframinces constamment agissantes : ils présentent, « en conserve », le produit de microcauses à peine sensibles, le résultat des interactions causales, ou «coïncidences » seulement concevables, qui interviennent à tout moment dans l'univers («En réalité, c'est le monde tout entier qui est fondé sur le hasard $\left.{ }^{27} »\right)$. Les 3 stoppages étalon donnent une forme visuelle à ce que la science ne peut mesurer, à ce qui est impondérable et partout présent. Le détail des ondulations des trois lignes épouse l'incommensurable, lui donne une figure précise. En conséquence, la reproduction de ces ondulations, résultat de la coïncidence de causes inframinces, ne peut avoir lieu que par duplication aussi exacte que possible (la règle épousant les courbes des fils), puisqu'il est impossible de réitérer à l'identique l'événement de la chute. Cette reproduction mécanique, à la règle, engendrera elle-même des différences inframinces, suspendant tout rapport d'original à copie par la multiplication de petites différences exactes : « Tous les "identiques” aussi identiques qu'ils soient,

25. Les définitions et exemples de situations inframinces sont regroupés dans les notes 1 à 46 de Duchamp (" Inframince ", in Marcel Duchamp, Notes, Paris, Flammarion, 1999, p. 21-47). Le " possible » est défini comme " infra mince " (note 1).

- 26. Man Ray compare L'Élevage de poussière à " un étrange paysage vu par un oiseau " ("Élevage de poussière ", in Ce que je suis et autres textes, Paris, Hoëbeke, 1998, p. 89).

27. Entretien de Duchamp avec Francis Roberts, "I Propose to Strain the Laws of Physics », cité par Bernard Marcadé in Marcel Duchamp, op. cit., p. 91. 
(et plus ils sont identiques) se rapprochent de cette différence séparative infra mince ${ }^{28}$. » Duchamp, réalisant le protocole exact des 3 stoppages étalon, inscrit précisément l'instant présent : le hasard suspend l'opposition entre neutralité et expressivité pour montrer le résultat d'un geste éminemment circonstancié, indissociable des causes infimes agissant à un moment donné.

Les agencements fortuits peuvent ainsi rencontrer les lois de la pataphysique d'Alfred Jarry, auteur bien connu de Duchamp, qui définit celle-ci comme « la science du particulier, quoiqu'on dise qu'il n'y a de science que du général. Elle [la pataphysique] étudiera les lois qui régissent les exceptions et expliquera l'univers supplémentaire à celui-ci ; ou moins ambitieusement décrira un univers que l'on peut voir et que peut-être l'on doit voir à la place de l'univers traditionnel ${ }^{29}$. » On peut expliquer la chute d'un fil par les lois générales de la gravité, qui valent pour tout objet solide ; mais on peut aussi en rendre compte par « les lois des exceptions », ancrées dans la considération singulière des coïncidences et des circonstances présidant au mouvement particulier de la chute et à la singularité des ondulations du mètre, déformé « à son gré ${ }^{30}$ ». Cette loi d'exception pataphysique ayant pour objet le hasard est une loi obtenue non par induction, mais par la multiplication et la répétition de situations échappant à toute loi ${ }^{31}$. La règle en bois, épousant la réalité, ne peut être fixée en amont, à l'image de cette mesure des mesures qu'est « l'étalon », mais en aval, à partir des sinuosités singulières d'un événement. La «science des exceptions », que viennent figurer les ondulations inframinces des trois fils, oblige à considérer le monde sous un angle radicalement autre, abandonnant le privilège accordé à l'induction, à l'homogénéité et à la généralisation pour l'observation multiple d'instants donnés dans un univers constamment changeant.

28. Marcel Duchamp, note 35, in Notes, op. cit., p. 33.

29. Alfred Jarry, Gestes et opinions du docteur Faustroll, pataphysicien, Paris, La Différence, 2010, p. 141.

- 30. L'expression " à son gré » qualifie le « hasard pur » du mouvement de la chute : « [...] mettre quelque chose sur une toile, sur un bout de papier, associer l'idée d'un fil droit horizontal d'un mètre de longueur tombant d'un mètre de hauteur sur un plan horizontal à celle de sa propre déformation, à son gré » (Pierre Cabanne, Entretiens avec Marcel Duchamp, op. cit., p. 81, nous soulignons). Cette expression peut évoquer, indirectement, la description du clinamen des atomes chez Lucrèce, qui dévient sponte sua, "spontanément, de son propre mouvement " (voir par exemple le deuxième paragraphe du livre IV du De natura rerum). Le clinamen est un exemple parfait d'écart inframince : les atomes qui tombent dans le vide dévient «à peine et le moins possible ", selon ce minimum physique le plus petit possible (" un tant soit peu », " juste assez pour qu'on puisse dire que leur mouvement se trouve modifié »), mais suffisamment réel pour déterminer un écart tel qu'il puisse être cause d'une déviation entraînant des chocs, des rencontres, des collisions, des attractions et répulsions, donc un monde produit par les combinaisons de la matière (voir le livre II du De natura rerum; la citation renvoie à la traduction d'Alfred Ernout dans l'édition " Les Belles Lettres ॥, Paris, 1947, p. 56-57). Le concept de clinamen apparaît dans les Gestes et opinions du docteur Faustroll, pataphysicien d'Alfred Jarry, au chapitre XXXIV, en lien avec une « machine à peindre, animée à l'intérieur d'un système de ressorts sans masse » (op. cit., p. 395-396).

- 31. Ce basculement de perspective est au centre de la pensée de Jarry : " La science actuelle se fonde sur le principe de l'induction : la plupart des hommes ont vu le plus souvent tel phénomène précéder ou suivre tel autre, et en concluent qu'il en sera toujours ainsi. D'abord ceci n'est exact que le plus souvent, dépend d'un point de vue, et est codifié selon la commodité et encore ! " (Gestes et opinions du docteur Faustroll, pataphysicien, op. cit., p. 142) 


\section{La roulette comme jeu de patience}

Marcel Duchamp, Obligation pour la roulette de Monte-Carlo $n^{\circ} 12,1924,31,2$ x 19,3 cm, New York, Museum of Modern Art (MoMA).

Parmi les ready-made rectifiés figure l'Obligation pour la roulette de Monte-Carlo, réalisée en 1924, au moment où Duchamp se consacre plus intensément aux tournois et compétitions d'échecs. Il s'agit d'une lithographie en couleurs, faite à partir d'une obligation réelle reproduisant le tapis vert d'un jeu de roulette, assortie d'une photographie de Duchamp 
prise par Man Ray, avec pour fond un jeu de mots indéfiniment répété en petites italiques vertes («moustiques domestiques demistock»). La tête de Duchamp apparaît au centre d'une roulette, le visage recouvert de mousse à raser et les cheveux dressés en cornes, tel un diable domestique du matin ou un descendant du dieu Pan, ici auréolé de la roulette. La valeur de l'obligation est indiquée en lettres capitales : «obligation de cinq cents francs, au porteur $20 \%$ », soit une créance de cinq cents francs avec un intérêt de $20 \%$ pour le détenteur de l'obligation. Le calembour, " moustiques domestiques demistock », entre en résonance avec les autres éléments de l'obligation : Duchamp, piqué par le jeu, confectionne une obligation domestique (signée par Duchamp dans le rôle d'« Un Administrateur » et Rrose Sélavy en «Président du Conseil d'Administration») dont la finalité réside, comme nous le verrons, dans l'équilibre du partage (« demistock») entre les gains et les pertes.

Ce ready-made se distingue des autres, non parce qu'il serait « rectifié » ou « aidé » (c'est le cas d'un grand nombre de ready-made ${ }^{32}$ ), ni parce qu'il est édité en une série de trente-cinq coupons (les ready-made seront réédités en série limitée dans les années 1960), mais bien parce qu'il a une valeur indéniablement utilitaire : Duchamp souhaite vendre les obligations afin d'obtenir un capital suffisant pour mettre à l'épreuve, par le jeu, des séries de calculs probabilistes élaborés pendant plusieurs mois à Monte-Carlo, à partir de sa propre pratique du jeu de hasard et de l'étude des combinaisons des numéros sortis ; autrement dit, il s'agit de se donner les moyens d'appliquer une martingale personnelle. Pour faire connaître ce projet d'obligation "domestique », un communiqué est publié dans The Little Review, revue new-yorkaise consacrée à l'avant-garde littéraire et artistique. Cette annonce décrit l'obligation comme une opération rentable, non pas au regard des intérêts qu'elle propose, mais pour la valeur que confère à cette émission la signature de Duchamp.

Cette lubie apparente, qui retient Duchamp à Monte-Carlo pendant quelques mois, s'intègre parfaitement à la mécanique duchampienne, à condition de souligner, là encore, les déplacements, les écarts, les entreprises de neutralisation et de suspension qui permettent à Duchamp de jouer sans jouer, d'adhérer à distance, par un équilibre savant entre valeurs contraires.

Ce qui ressort immédiatement à la lecture des lettres et des notes de Duchamp est l'absence totale d'investissement passionnel : le jeu se présente comme un objet de pensée procurant une sorte de distraction intellectuelle amusante, «d'une monotonie délicieuse » et dépourvu de « la moindre émotion ${ }^{33} »$. Duchamp ne joue pas vraiment, mais semble jouer à jouer, instaurant une distance qui court-circuite la capacité du jeu à absorber les esprits. Cette distance, Duchamp la maintient méthodiquement en considérant le jeu comme l'occasion de calculs de probabilités sans fin, prétexte à élaboration de tactiques contingentes et provisoires, toujours reconduites.

32. Cf. "À propos des ready-made », in Duchamp du signe, op. cit., p. 191-192.

33. "Lettre de M. Duchamp à F. Picabia, 17 avril 1924 ", in Francis M. Naumann et Hector Obalk (éd.), Affectionately, Marcel: The Selected Correspondence of Marcel Duchamp, Gand et Amsterdam, Ludion Press, 2000, p. 144. 
Aborder le hasard de la roulette à travers les calculs, dans une perspective rationnelle, est une position somme toute assez commune : les systèmes de martingale promettant au joueur de remporter la mise sont aussi vieux que les jeux mêmes, et les jeux de hasard ont souvent servi de support théorique et empirique aux développements des calculs de probabilités ${ }^{34}$. Mais Duchamp utilise la martingale en la vidant de son sens. Car celle-ci n'a jamais éliminé la passion du jeu, bien au contraire : elle ne fait que la déplacer en entretenant l'illusion d'une rationalité globale au sein d'une suite de chiffres qui ne peut être pensée que de manière probabiliste. Duchamp n'attribue pas à la martingale la rationalité suprême qu'on lui accorde ( $\mathrm{La}$ martingale n'a pas d'importance. Elles sont toutes bonnes et mauvaises $\left.{ }^{35} »\right)$. Elle ne doit pas lui permettre de gagner à coup sûr, donc de vaincre le hasard, mais simplement de ne pas perdre, donc d'arriver à maintenir un équilibre constant entre les pertes et les gains :

J'ai commencé à jouer : et la lenteur des progrès en plus ou moins est une épreuve de patience. Je me promène dans l'égalité, où je marque le pas de façon inquiétante pour ladite patience. Mais enfin faire cela ou autre chose. Je ne suis ni ruiné ni milliardaire et ne serai jamais ni l'un ni l'autre ${ }^{36}$.

On comprend ce qui peut attirer Duchamp dans le projet de martingale : toute martingale est un défi au hasard et répond à l'ambition de trouver une logique sous-jacente à ce qui échappe à toute logique, au désir de faire apparaître une rationalité dans un jeu conçu pour l'exclure, et ce, non en trichant ou en brisant les règles, mais en les respectant. Duchamp dépouille cependant la martingale de toute promesse pour en faire un simple outil de conservation des mises (« je ne suis ni ruiné ni milliardaire »). Il entreprend donc de jouer avec « un esprit mécanisé contre une machine. Rien de romantique dans l'affaire, pas plus que de chance ${ }^{37}$. » Jouer avec « un esprit mécanisé » ne suppose pas simplement de calculer (le calcul peut entretenir la passion) mais, plus précisément, de voir la machine comme une simple machine, comme une mécanique sans mémoire. Mécaniser l'esprit revient à le dépouiller de cette attitude superstitieuse qui repose, comme le dit Émile Borel dans les analyses qu'il consacre aux jeux de hasard, sur « la croyance anthropomorphique à la mémoire et à la conscience de la pièce de monnaie ${ }^{38} »$ : le joueur pris par le jeu se comporte face à la machine comme face à un être doué de mémoire, il estime la probabilité du coup suivant à partir des coups précédents et suppose donc, à tort, que les tirages ne repartent pas de zéro mais s'accumulent les uns aux autres. Mécaniser l'esprit suppose de ne voir dans la roulette que ce qu'elle est,

34. Cf. Louis Bachelier, Le Jeu, la Chance et le Hasard, Paris, Flammarion, 1914, p. 6 et suivantes. Rappelons également que le narrateur du roman de Dostoïevski, Le Joueur, évoque les personnes qui jouent « le crayon à la main ", très perturbées lorsque les chiffres se présentent en séries régulières.

35. "Lettre de M. Duchamp à F. Picabia, 17 avril 1924 », in Affectionately, Marcel, op. cit., p. 144.

36. "Lettre de M. Duchamp à J. Doucet, juin 1925 », ibid., p. 151.

37. "Lettre de M. Duchamp à Stettheimer, mars 1925 », ibid., p. 150.

38. Émile Borel, Le Hasard, chapitre II : "Les lois du jeu de pile ou face ", Paris, Librairie Félix Alcan, 1920, p. 19. 
une mécanique simple produisant une succession de coups parfaitement indépendants les uns des autres.

La martingale élaborée par Duchamp n’est donc pas un système de calculs permettant de lutter contre le hasard, mais un projet qui cherche à faire se rencontrer les opposés, à atteindre ce point d'équilibre qui incarne le principe duchampien de « cointelligence des contraires ${ }^{39}$ » : non pas gagner (c'est impossible), mais rationaliser juste assez pour ne pas perdre. Ce qui suppose, pour reprendre une distinction établie par Poincaré, de renoncer à toute « probabilité subjective », établie à partir des seules séries qui retiennent notre attention, pour une « probabilité objective », considérée à l'échelle globale d'un jeu « qui se prolonge longtemps ${ }^{40}$ ». La temporalité diffère : au coup par coup relançant la chance ou la malchance et focalisant la tension sur les suites de nombres remarquables (par exemple sur la sortie du rouge six fois de suite), se substitue la considération globale de l'ensemble des coups sur un laps de temps relativement long, au cours duquel il s'agit non tant d'avancer ou de reculer que de «marquer le pas », donc de faire du surplace. La somme que Duchamp espère recevoir doit lui permettre de jouer assez longtemps pour voir s'équilibrer les rouges et les noirs, les pertes et les profits, rejoignant cet équilibre global que présente toute suite de phénomènes aléatoires indépendants. Duchamp illustre ce point d'équilibre en expliquant qu'en « introduisant plus de chance du côté des échecs et en réduisant le facteur chance du côté de la roulette, ces deux activités peuvent se rejoindre d'une certaine manière ${ }^{41}$ ». Les échecs ne sont pas un jeu de hasard, mais bien l'exemple même d'un jeu parfaitement rationnel, ou « jeu à information parfaite » dans la théorie des jeux ; mais plus la partie progresse, plus les stratégies des joueurs sont complexes, moins il est possible d'anticiper exhaustivement le déroulement de la partie de l'adversaire : les coups peuvent alors devenir fortuits, croisant deux séries de faits (les stratégies de chaque joueur) indépendantes l'une de l'autre $^{42}$. Inversement, si la roulette est le jeu de hasard par excellence, il est possible, en jouant, de viser ce stade au sein duquel les rouges et les noirs s'équilibrent et annulent, par cumulation, leurs effets respectifs en termes de pertes et de profits - équilibre qui, sans sortir du jeu et de ses règles, suspend

39. Cette expression apparaît dans la note 185 de Duchamp (in Notes, op. cit., p. 112).

- 40. Voir les "Réflexions sur le calcul des probabilités " de Poincaré : "On comprend que, sans rien savoir

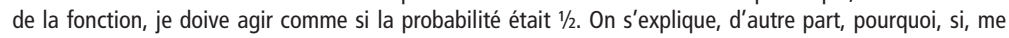
plaçant au point de vue objectif, j'observe un certain nombre de coups, I'observation me donnera à peu près autant de coups noirs que de coups rouges. Tous les joueurs connaissent cette loi objective ; mais elle les entraîne dans une singulière erreur, qui a été souvent relevée, et dans laquelle ils retombent toujours. Quand la rouge est sortie, par exemple, six fois de suite, ils mettent sur la noire, croyant jouer à coup sûr ; parce que, disent-ils, il est bien rare que la rouge sorte sept fois de suite. En réalité, leur probabilité de gain reste 1/2. " (Henri Poincaré, L'Analyse et la Recherche, Paris, Hermann, 1991, p. 128)

- 41. Propos cités par Arturo Schwarz dans The Complete Works of Marcel Duchamp et rapportés par Bernard Marcadé dans Marcel Duchamp, op. cit., p. 269. On retrouve la même idée dans une lettre adressée à Jacques Doucet, datée du 16 janvier 1925 : « Je voudrais avoir forcé la roulette à devenir un jeu d'échecs » (in Duchamp du signe, op. cit., p. 270).

42. Cournot, réfléchissant aux conditions par lesquelles une suite d'événements s'inscrit, ou non, dans l'histoire, donc aux conditions de possibilité de l'historicité, établit une gradation allant des « registres d'une loterie publique » (dépourvus d'histoire) aux jeux d'échecs. Les combinaisons du jeu d'échecs font surgir le hasard au sein d'une rationalité globale : " [...] la détermination réfléchie du joueur se substitue aux hasards du dé, de manière pourtant à ce que les idées du joueur, en se croisant avec celles de l'adversaire, donnent lieu à une multitude de rencontres accidentelles " (Antoine-Augustin Cournot, Essai sur les fondements de nos connaissances et sur les caractères de la critique philosophique, Paris, Hachette, 1922, p. 460). 
son intérêt. Les échecs et la roulette peuvent ainsi se rejoindre en ce point d'équilibre précaire, impossible à fixer objectivement, où la rationalité des échecs se hasarde en " un coup » chanceux et l'aléa des tirages au sort se rationalise approximativement.

Duchamp transforme donc la roulette en un jeu de patience : il faut être capable de jouer assez longtemps pour maintenir l'équilibre constant, car « la lenteur des progrès en plus ou moins est une épreuve de patience ${ }^{43} »$; le jeu de hasard devient un exercice de discipline, une mise à l'épreuve de l'indifférence et de la patience dans le temps long de coups aléatoires qui se succèdent sans mémoire, donc sans histoire. Mais pour qui joue sans jouer vraiment, ou joue à jouer, sans passion

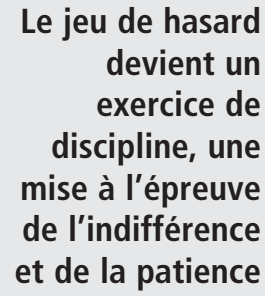

Le jeu de hasard devient un exercice de discipline, une mise à l'épreuve de l'indifférence et de la patience ni émotion, sans illusion d'aucune sorte, mais avec le souci de maintenir une forme d'économie de soi (et de sa mise), le jeu de hasard s'avère le plus ennuyeux des jeux : " Il fallait quelquefois attendre une demi-heure que les numéros apparaissent dans la succession des rouges et des noirs et les quelques semaines que je passai à Monte-Carlo furent si ennuyeuses que j'abandonnai bientôt, heureux de m'en tirer sans perte ${ }^{44}$. » Duchamp est, en quelque sorte, victime de son propre jeu, ou plutôt de son absence de jeu : l'indifférence amusée devient, à la longue, ennui désabusé. Il quitte au bout de quelques mois la table de jeu pour revenir aux stratégies autrement plus complexes des cases de l'échiquier.

Comme dans 3 stoppages étalon, le hasard ne produit ses effets qu'à condition d'être dépouillé de son caractère chanceux ou malchanceux, et ne fait sens que par les mouvements et associations créés dans la pensée (associer les courbes visibles avec les causes inframinces de la chute dans 3 stoppages étalon, mettre en rapport le hasard de chaque coup avec les calculs probables dans le jeu de la roulette). De ce projet de martingale sans promesse, boussole approximative permettant de « se promener dans l'égalité », il reste ce tour de force : en déshumanisant le jeu de hasard, en le mécanisant, Duchamp transforme la roulette en jeu de patience et intègre cette activité à l'ensemble de son œuvre, effaçant les frontières entre « art » et « non-art » au profit d'un « art de vivre » mobilisant les mêmes principes, quel que soit le domaine concerné.

\section{L'Erratum musical :}

\section{imprimer de la musique sur fond d'oubli}

L'Erratum musical de 1913 est présenté dans les notes de la Boîte verte ${ }^{45}$ sous forme de partition et de texte à chanter. Le procédé est relativement simple : il s'agit de tirer au sort des bouts de papier indiquant chacun une note, puis de les inscrire sur une portée en respectant l'ordre de tirage,

43. "Lettre de M. Duchamp à J. Doucet, juin 1925 », in Affectionately, Marcel, op. cit., p. 151.

44. Marcel Duchamp, "À propos de moi-même ", in Duchamp du signe, op. cit., p. 229.

45. Le procédé, ainsi que la partition qui l'accompagne, est présenté dans la Boîte verte (in Duchamp du signe, op. cit., p. 52-53). 
Marcel Duchamp, Erratum musical, 1913, dessin au crayon et encre noire sur papier à musique, 317 x $482 \mathrm{~cm}$, Paris, musée national d'Art moderne - Centre Pompidou.

sans précision de rythme ni de tempo. Le tirage au sort est effectué trois fois, de manière à produire trois mélodies, qui sont écrites sur « trois partitions différentes ». Chaque mélodie est elle-même constituée de vingt-cinq notes, dont aucune ne se répète - on suppose donc que chaque papier est mis de côté après avoir été tiré au sort, et que le nombre de possibilités à chaque tirage va décroissant. La partition doit être chantée par «trois personnes »; dans la version écrite par Duchamp et reproduite dans la Boîte verte, les noms de « Marcel » et de ses sœurs, « Yvonne » et «Magdeleine », sont inscrits sur la partition, au-dessus de chaque partie. Les notes sont chantées sur les mots suivants, décomposés en vingt-cinq syllabes (une syllabe par note) : « faire une empreinte marquer des traits une figure sur une surface imprimer un sceau sur cire». Ce texte, sorte de ready-made textuel, est la définition du verbe « imprimer », extraite d'un dictionnaire. Telles qu'elles sont présentées, les trois mélodies peuvent être enchaînées les unes aux autres ou chantées simultanément. En observant la partition, on peut également remarquer que les notes écrites en clé de fa sont beaucoup plus présentes dans la partie réservée à « Marcel » que dans celles des deux sœurs, ce qui laisse supposer que les résultats du tirage ont été quelque peu modifiés de manière à rendre les données du hasard réalisables pour une voix basse. L'Erratum musical est bien, comme le dit Bernard Marcadé, une « composition musicale à usage domestique ${ }^{46}$ », semblable en cela à l'Obligation pour la roulette de Monte-Carlo : ce n'est pas une idée restée à l'état de projet, mais un procédé défini et réalisé sur du papier à musique, les partitions étant personnellement attribuées afin d'être chantées en famille. 
Comme 3 stoppages étalon et Roue de bicyclette, réalisés la même année (1913), Erratum musical participe pleinement à la mise en place des principes de l'esthétique duchampienne : l'absence d'émotion (la musique est inexpressive), l'annulation du goût (choisir les notes au hasard) et l'indifférence aux normes (la musique n'est pas composée). Il nous semble cependant que partir de ces principes, pour favoriser une lecture systématique de l'ensemble de l'œuvre de Duchamp, nous éloigne de la spécificité de cette partition et des questions qu'elle soulève sur la nature même de la composition et de l'écoute musicale. Le plaisir est lié autant à la performance qu'aux réflexions qu'elle suscite sur les mécanismes de la composition musicale, les rapports de tension entre la faute (Erratum) et la norme, la conservation par impression mécanique et l'impossibilité de toute mémorisation.

Pour réaliser l'Erratum et chanter les données du hasard, on doit nécessairement inscrire les résultats du tirage sur une partition qui enregistre les données une à une. Cette musique ne peut être imaginée avant d'être jouée, elle suppose donc une trace écrite, non comme l'aboutissement d'une projection ou d'une réflexion préalable, mais comme origine non expressive d'une musique inscrite au gré des tirages. De même qu'on ne peut savoir, en lâchant trois fils, quelles seront leurs formes immobilisées sur la toile, on ne peut non plus prévoir les lignes des trois mélodies issues du tirage au sort et fixées sur le papier. Le papier à musique est cette « cire » évoquée dans le texte chanté, sur laquelle chaque note, tirée d'un chapeau, vient s'imprimer directement, sans passer par aucune opération de contrôle ou de choix : il conserve la trace d'une opération de hasard qui est, comme dans 3 stoppages étalon, indissociable du résultat (je ne peux écouter l'Erratum sans penser au tirage au sort qui lui sert de matrice).

L'écriture aléatoire donne une musique imprévisible et inexpressive : l'Erratum ne peut s'écouter comme une phrase musicale, une mélodie dont on pourrait suivre le mouvement. Les notes tombent dans l'instant, une à une, sur le même plan, sans être soutenues par la continuité des relations harmoniques ni par la périodicité d'un rythme quelconque ; une note chasse l'autre, ne résonne avec la précédente que le temps d'une rémanence ponctuelle ${ }^{47}$. La grande disparité et la diversité des intervalles rendent difficile

47. Il y a, à notre connaissance, deux enregistrements disponibles de la version chantée du premier Erratum musical : I'une dans The Music of Marcel Duchamp (Block-Paula Cooper Gallery, 1991, interprété par I'ensemble SEM), I'autre dans The Creative Act (Sub Rosa, 1994, interprété par Marianne Pousseur, Jean-Luc Plouvier et Lucy Grauman). On peut écouter la première version sur le site "ubuweb : sound " : http://www. ubu.com/sound/duchamp.html. Ces deux interprétations sont très différentes l'une de l'autre : la première (1991) fait entendre chaque voix séparément, puis les trois voix ensemble, six fois de suite. Le tempo est constant, chaque note est chantée de manière appuyée, avec une durée identique. Cette interprétation rend parfaitement l'étrangeté de cette musique qui, en dépit des nombreuses répétitions et de l'homogénéité de I'ensemble, se dérobe à la mémoire. La seconde interprétation (1994) est beaucoup plus expressive et introduit des différences qualitatives importantes d'une reprise à l'autre : les trois voix sont superposées et le texte est chanté quatre fois, la première fois de manière soutenue, la deuxième legato, la troisième détachée et piano, la dernière sur un tempo plus rapide, accompagnée d'un jeu de grelots. Ainsi interprétée, la partition se trouve en quelque sorte re-subjectivée et se rapproche du divertissement musical. 
la mémorisation du chant par l'auditeur : les notes aléatoires sont sans temporalité, non simplement en raison de l'absence de rythme défini, mais parce qu'elles se dérobent à toute saisie en fonction de l'avant et de l'après. La mélodie se déroule tout en faisant du surplace, et la répétition du texte, chanté trois fois, ne crée aucun approfondissement et ne change rien à la compréhension de l'ensemble. Erratum musical s'écoute sur fond d'oubli immédiat. Ce qui ne peut s'anticiper ni se mémoriser peut cependant se conserver par impression immédiate sur cette surface de « cire » qu'est la partition. L'Erratum actualise donc une différence essentielle entre mémoire et conservation (par impression) qui rappelle, indirectement, le passage célèbre du mythe de Theuth dans le Phèdre de Platon. Ce mythe, rapporté par Socrate, expose la différence entre la mémoire vive et la conservation de la trace écrite dans l'acquisition et la préservation d'un savoir. L'écrit apparaît dans ce dialogue comme l'envers de la mémoire véritable : à l'acte de mémoire, qui procède d'un effort et d'une recherche interne, vivant car toujours repris, s'opposent les « empreintes étrangères » de l'écriture, délivrant un savoir que le lecteur n'acquiert qu'en apparence, de l'extérieur. L'écriture, loin d'enrichir la mémoire, « produira l'oubli » dans l'âme de ceux qui s'en remettent à la seule conservation de la trace écrite et cessent « d'exercer leur mémoire 48 ». Comme les « empreintes étrangères » d'une tablette de cire, la partition aléatoire de l'Erratum musical se conserve hors de la mémoire.

Le registre de l'impression mécanique apparaît aussi dans le titre. Un erratum est une faute d'impression, signalée comme telle dans la liste éventuelle des errata accompagnant une édition. Il suppose une norme préalable permettant d'identifier la faute, une règle au regard de laquelle un terme est jugé incorrect. Le titre, Erratum musical, souligne donc le caractère particulièrement dissonant de cette musique qui s'apparente, pour l'oreille habituée aux règles de l'harmonie tonale, à une faute musicale généralisée. Car l'Erratum, contrairement à ce que l'on pourrait penser, n'est pas un objet totalement « hors norme », qui annulerait toute référence sous-jacente à la composition. En dépit du caractère aléatoire de la méthode, la pièce sonne de manière inhabituelle sans être pour autant chaotique. Le résultat présente en effet une certaine homogénéité, due à la présence du texte énoncé de manière identique à chaque voix ainsi qu'à la durée régulière de chaque son. Le note à note extrêmement appuyé crée un principe d'ordre tout aussi manifeste à l'écoute que l'incongruité des intervalles. Les trois mélodies sont différentes et semblables à la fois, comme les trois fils d'un mètre de long des 3 stoppages étalon. Là encore, la répétition est un élément essentiel de la démarche (une partition qui serait constituée d'une seule série de vingt-cinq notes tirées au sort ne serait pas un Erratum). Car seule la répétition permet d'associer indissociablement la différence irréductible de chaque mélodie et l'homogénéité de l'ensemble : comme les trois fils, les trois mélodies sont chacune imprédictibles et pourtant équivalentes ; elles échappent à la mémoire et à la compréhension, mais appartiennent pourtant à un même plan d'ensemble. 
Nous souhaitons, en conclusion, comparer Erratum musical à son homologue littéraire le plus proche, écrit quelques années plus tard : le « poème dadaïste », dont Tristan Tzara décrit le principe de fabrication dans le «Manifeste sur l'amour faible et l'amour amer ${ }^{49} »$ (1920). Les instructions de Tzara sont suivies d'un poème qui a valeur d'exemple, exactement comme la partition de l'Erratum. Si le geste est semblable (tirer des mots ou des notes d'un chapeau, puis les aligner tels quels, les uns à la suite des autres, sur une page ou une portée), l'indétermination est beaucoup plus grande dans le poème de Tzara que dans l'Erratum de Duchamp. En raison du nombre de mots, qui dépend de la taille de l'article choisi, de leur diversité et de l'absence de syntaxe, le poème est éclaté et totalement incohérent. En comparaison, le champ des possibilités dans l'Erratum semble beaucoup plus restreint ${ }^{50}$ et se traduit, à l'écoute, par l'instabilité particulière de certains accords, parfois moins discordants que déviants, pas tout à fait à leur place. Aussi pouvons-nous penser que Duchamp choisit le singulier erratum, et non le pluriel errata plus commun, pour rappeler, en plus de la référence directe à l'impression mécanique, le participe passé du verbe latin errare : errer, perdre son chemin, faire fausse route, douter. L'Erratum est le produit d'une errance de la ligne mélodique, qui sonne comme une faute d'impression, non respectueuse de la norme sans pour autant ignorer celle-ci. Le hasard du poème de Tzara a la provocation du rire, l'éclat de l'absurde. Le « hasard en conserve » de Duchamp joue sur un autre registre, subtilement corrosif : les errances de la mélodie, les lignes «possibles» des 3 stoppages étalon et l'égalité « en plus ou moins » de la roulette viennent figurer l'inframince séparant chaque donnée factuelle de la norme qui la sous-tend et qu'elle vient éroder, insidieusement, précisément.

Sarah Troche

professeur de philosophie, lycée Jules-Ferry, Conflans-Sainte-Honorine

49. Tristan Tzara, Sept Manifestes dada, Paris, Jean-Jacques Pauvert, 1963, p. 62-64.

50. En observant la partition, on constate que les notes sont réparties au sein d'un ambitus allant du do en clé de fa (deuxième interligne, dans la partie de Marcel), au fa de la ligne supérieure en clé de sol (dans la partition d'Yvonne), ce qui correspond à une série de trente notes possibles (en comptant les demi-tons, présents sur la partition). 